\title{
"A Double-Edged Sword": A Case Report on Liver Injury Side-Effect of Anti-Tuberculosis Drug on Liver Tuberculosis Patient
}

Azlina Ibrahim ${ }^{1}$, Alvin Oliver Payus ${ }^{2 *}$

\author{
${ }^{1}$ Department of Medicine, \\ Hospital Kuala Lumpur, \\ Kuala Lumpur, Malaysia \\ 2 Department of Medicine-Based Discipline, \\ Faculty of Medicine and Health Sciences, \\ Universiti Malaysia Sabah, \\ Kota Kinabalu, Sabah, Malaysia
}

\section{*Corresponding author's email: dralvinpayus@ums.edu.my}

Received: 18 February 2019

Accepted: 17 October 2019

Keywords:

hepatic tuberculosis, AIDS,

tuberculosis, biopsy

\section{ABSTRACT}

Hepatic involvement in extra-pulmonary tuberculosis (TB) is rare, even in the endemic area. It has a high mortality rate as it can easily be misdiagnosed due to its rarity and nonspecific presentations, and the treatment can be challenging for its hepatotoxic side-effect. A 55year old man who was newly diagnosed with AIDS and pulmonary TB which complicated with antiTB-induced transaminitis, presented with a few weeks history of fever and persistent diarrhoea. It was initially treated as microsporidia infection but the symptoms persisted despite given antiparasitic agent for more than a week. He was subjected to computed tomography (CT) scan of the abdomen and noted multiple hypoechoic lesion at multiple segments of the liver, which later confirmed to be liver TB by liver biopsy. As he could not tolerate Akurit-4 (Rifampicin 150 mg, Isoniazid $75 \mathrm{mg}$, Pyrazinamide $400 \mathrm{mg}$ and Ethambutol $275 \mathrm{mg}$ ), the second-line treatment was given instead. He is currently well on regular clinic appointment. The objective of this case report is to share the rare occurrence of hepatic TB and the difficulty to treat it as the hepatotoxic effect of anti-TB medications complicate the liver damage due to the infection.

\section{INTRODUCTION}

Tuberculosis (TB) commonly affects the pulmonary system due to the nature of the organism that favours the high oxygen tension environment. Rarely, it also infects the liver which has lower oxygen concentration. This can occur with or without pulmonary involvement, and can either diffusely be 
disseminated over the liver, or locally affect a single lobe 1 . Hepatic TB is relatively a curable disease. However, the delay in diagnosis and treatment can lead to fatal consequences ${ }^{2}$. Furthermore, the treatment has a side effect that causes liver injury which synergized the damage caused by the infection itself. This rare extra-pulmonary TB is the main focus of discussion in this case report.

\section{CASE PRESENTATION}

A 55-year old male who is newly diagnosed with AIDS presented with a history of fever and persistent diarrhoea for a few weeks. Onemonth prior to the presentation, he was diagnosed with pulmonary tuberculosis (TB) started on 3 tablets a day of oral Akurit-4 (Rifampicin $150 \mathrm{mg}$, Isoniazid $75 \mathrm{mg}$, Pyrazinamide 400 $\mathrm{mg}$ and Ethambutol $275 \mathrm{mg}$ ), which was appropriate to his body weight. However, he developed drug-induced hepatitis whereby his alanine aminotransferase went up to $100 \mathrm{IU} / \mathrm{L}$, therefore second-line medication was initiated which include intramuscular streptomycin, oral ethambutol and moxifloxacin. At that time, his CD4 counts were 15 cells $/ \mu \mathrm{L}$ and the viral load of 127,000 copies/mL. Currently, upon presentation, he was febrile and severely dehydrated requiring aggressive fluid resuscitation and inotropic support. He was empirically treated for microsporidia infection and was planned for 3-weeks of oral albendazole. He was also restarted on oral Akurit- 4 as his liver function has normalized. However, he again developed marked transaminitis where his ALT jumped up four times from upper limit of normal after five days of restarting the medication which was then withheld and was changed back to second-line medications. Despite more than a week of oral albendazole, he still has persistent diarrhoea and intermittent fever. Blood cultures were negative. In view of persistent gastrointestinal symptoms with multiple spikes of temperature, CT scan of the abdomen was done and noted hypodense liver lesion in segment IV which suggestive of early liver abscess formation (Figure 1).

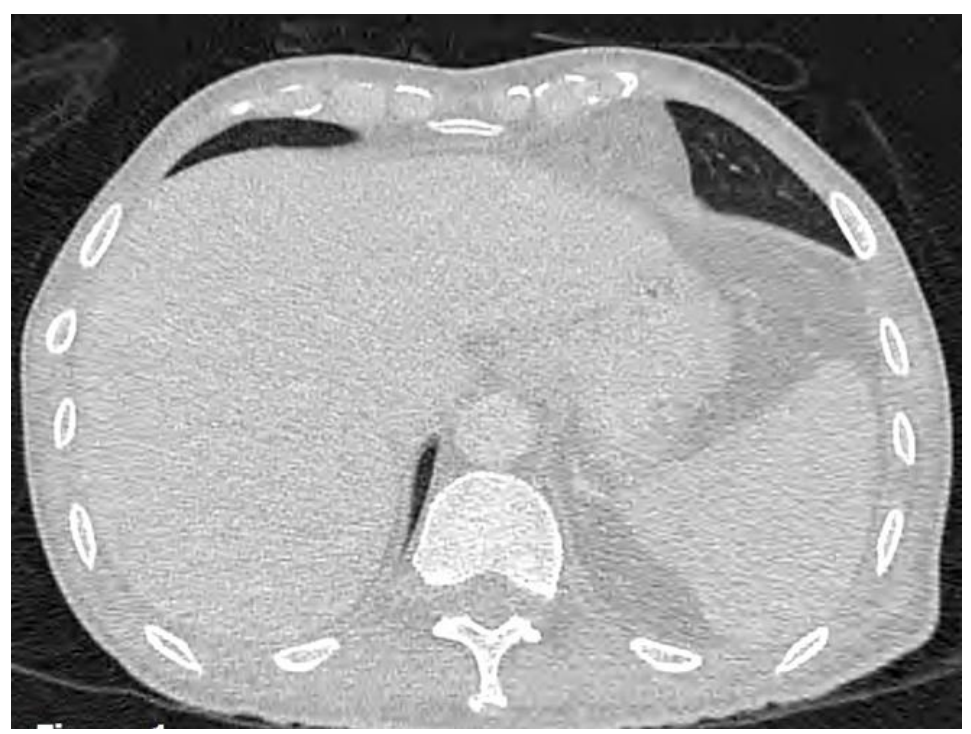

Figure 1 CT scan of the liver showed the hypodense area in segment IV likely represent liver abscess

He was then empirically treated for pyogenic liver abscess. A serial abdominal ultrasound showed multiple new hypoechoic lesions on other segments of the liver (Figure 2). As he was radiologically worsened, this raised the possibility of therapeutic failure. 


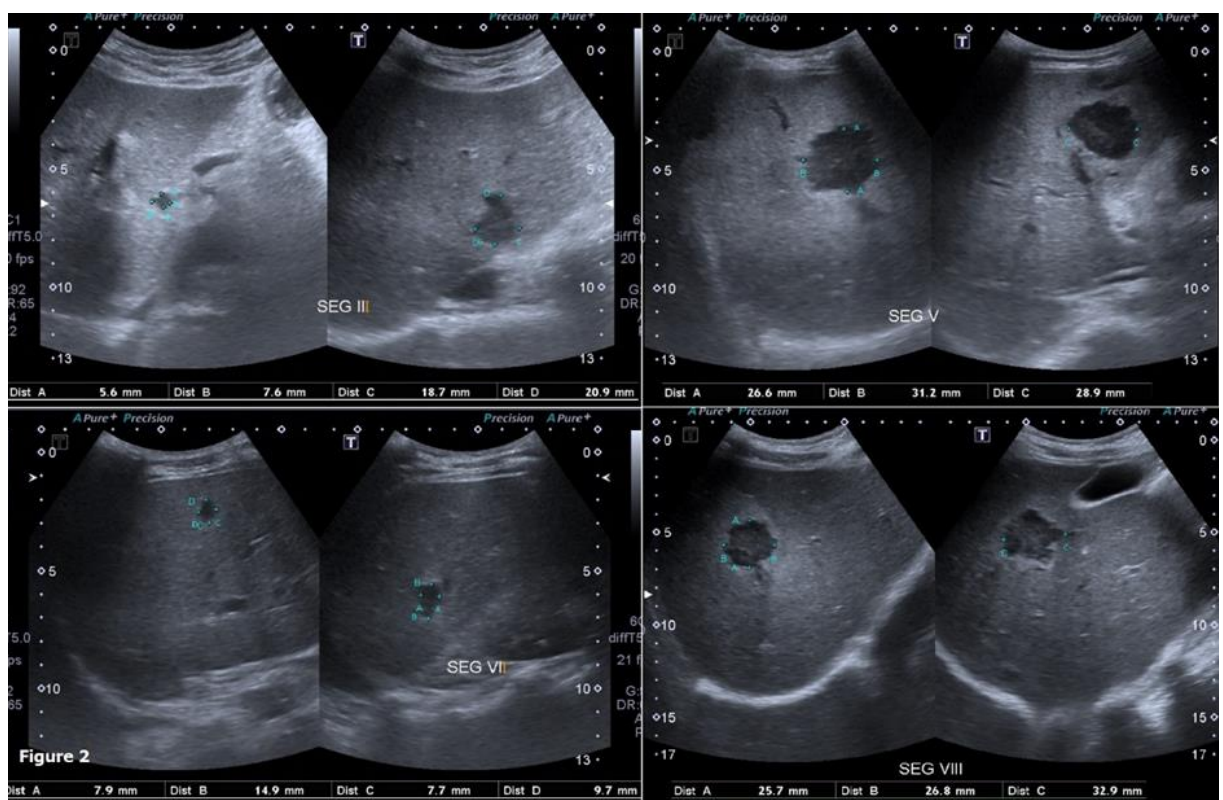

Figure 2 Ultrasound of the hepatobiliary system showed hypoechoic lesion over the segment II (left above), segment V (right above), segment VII (left bottom), segment VIII (right bottom)

He has then proceeded with a liver biopsy and it showed chronic granulomatous inflammations (Figure 3) with scattered acidfast bacilli organism upon special stain with Ziehl-Neelsen (Figure 4) which suggestive of liver TB. The treatment with second-line anti-TB was continued, and oral isoniazid was added on day 42 of treatment in which he tolerated. He completed the treatment for a total of 1-year with 2-months of intensive phase which consist of all four medications, followed by ethambutol and isoniazid for maintenance. His liver function has been normal and static to date.

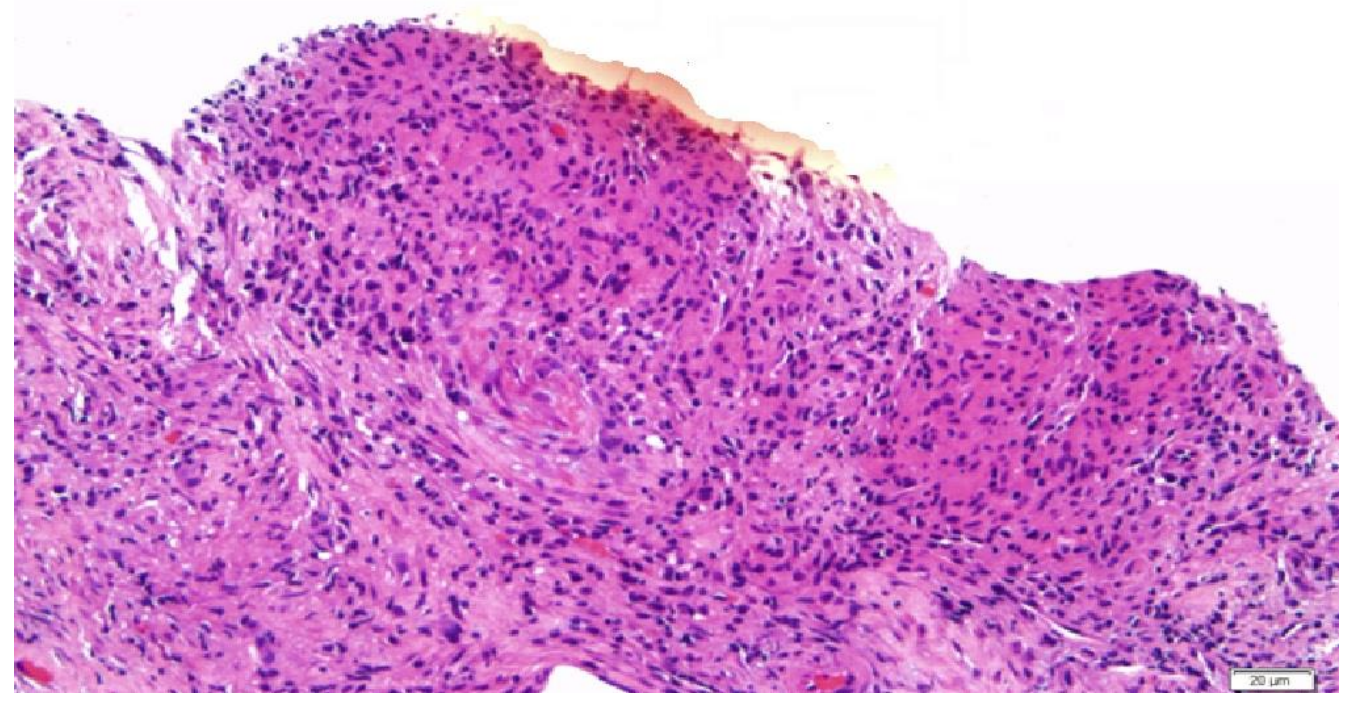

Figure 3 Histopathological examination of the liver tissue biopsy showed scattered granulation tissue with mixed inflammatory cells, predominantly histiocytes and occasional neutrophils. 


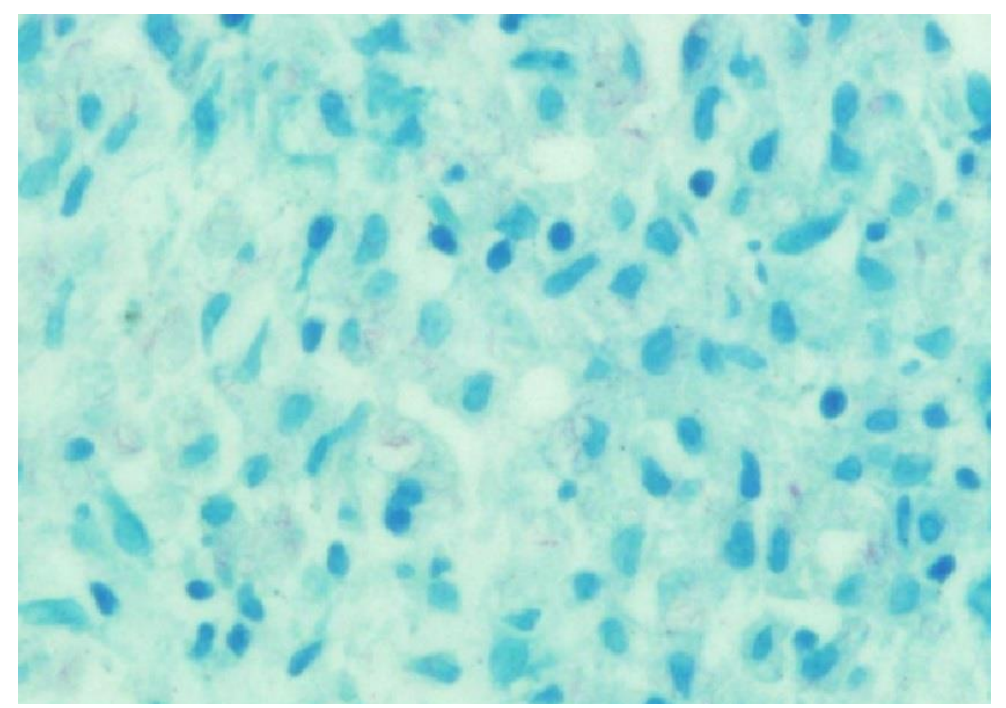

Figure 4 Microscopic examination under special staining showed scattered acid-fast bacilli organism which stained faint pink on Ziehl-Neelsen

\section{DISCUSSION}

Hepatic tuberculosis (TB) is rare which accounts only $1 \%$ of all TB infections ${ }^{1}$ and has a clinical presentation that highly mimics other common liver diseases such as pyogenic abscess, primary tumours and others. Classically it can present in three forms, and the commonest one is diffuse hepatic involvement seen along with pulmonary or miliary infection. This is seen in more than $50 \%$ of cases and is due to the haematogenous spread of mycobacteria via the hepatic artery. Patients are often asymptomatic despite the diffuse involvement of the liver. The second form is also known as granulomatous hepatitis, which is a diffuse hepatic infection without recognizable pulmonary involvement. The third and rarest form presents as a local tuberculoma'. Although hepatic TB relatively is a curable disease, it is associated with a high mortality rate. This is because hepatic TB lacks typical clinical presentations and diagnostic imaging features that make it easily misdiagnosed and causing delayed in treatment which lead to fatal consequences ${ }^{2}$. Acute liver failure secondary to TB infection was also reported but only in a few cases and most are associated with miliary $\mathrm{TB}^{3}$.
The common manifestations of hepatic TB are hepatomegaly, fever, abdominal pain and weight loss. Less commonly, it can also present with ascites, jaundice and splenomegaly. Patient with local hepatic TB may present with abdominal pain and jaundice while miliary hepatic TB may present with acute respiratory symptoms such as cough and sputum production ${ }^{4}$. Patient with elevated liver transaminases reflected the involvement of liver parenchyma whereas elevated alkaline phosphatase and gammaglutamyl transferase suggestive of biliary tree involvement. However, our patient had hepatitis secondary to anti-TB and have cause a delay in the diagnosis of hepatic TB.

Radiological investigations such as ultrasound, computed tomography and magnetic resonance imaging are useful diagnostic tools. Liver tuberculoma appears as a non-enhancing low-density centre, owing to caseation necrosis with a slightly enhancing peripheral rim which corresponded to surrounding granulation tissue ${ }^{5}$. The contrastenhanced ultrasonography may be helpful in differentiating the diagnosis of hepatic TB from other hepatic focal lesions ${ }^{6}$. However, the most specific test is liver biopsy with mycobacterial culture. The demonstration 
of acid-fast bacilli on special staining and caseous necrosis on biopsy can be very useful pathological tools but their absence cannot exclude the diagnosis.

Apart from the diagnostic difficulty, another major challenge in managing hepatic $\mathrm{TB}$ is the risk of hepatotoxicity that can be induced by anti-TB drugs. The incidence of antiTB induced hepatoxicity is about $24.6 \%$, with almost half occur within the first 2 weeks ${ }^{7}$. Chen $\mathrm{HC}$ et al. (2003) recommended treating the patient with hepatic TB with standard regimens despite the potential hepatotoxicity of these drugs, provided that the other cause of the liver injury must be excluded ${ }^{8}$. However, our patient developed transaminitis from the first line anti-TB after a few days of intensive phase, which have masked the liver injury caused by the infection. Therefore, he is not a suitable candidate for the first-line anti-TB treatment, especially oral pyrazinamide. But he did tolerate oral isoniazid and manage to take it for 1-year.

\section{CONCLUSION}

In conclusion, this case report demonstrates the double-effect of the anti-TB drug in treating hepatic TB where in one hand it has good therapeutic value but in the other hand it also has a hepatotoxic side effect that synergizes the damage caused by the infection. This side effect can mask the liver involvement and results in diagnosis delay. Therefore, one needs to suspect liver infiltration for patients with disseminated TB who had deranged liver function secondary to anti-TB drugs and initiate the proper diagnostic investigations and effective treatment promptly in order to save lives.

\section{ACKNOWLEDGEMENTS}

The authors would like to thank the patient for giving his consent and cooperation in relation to the writing of this case report. The author would also like to thank the Director General of Ministry of Health of Malaysia for his permission to publish this article.

\section{CONFLICT OF INTEREST}

The authors declare that they have no competing interests in publishing this case.

\section{CONSENTS}

Written informed consent was obtained from the patient to publish the case with its related pictures. A copy of the written consent is available for review by the Chief Editor.

\section{REFERENCES}

1. Sonika U, Kar P. (2012). Tuberculosis and liver disease: Management issues. Tropical Gastroenterology 33 (2): 102 - 106.

2. Chong VH. (2008). Hepatobiliary tuberculosis: a review of presentations and outcomes. Southern Medical Journal 101 (4): $356-361$.

3. Jain D, Aggarwal HK, Jain P, Pawar S. (2014). Primary hepatic tuberculosis presenting as acute liver failure. Oxford Medical Case Reports 2014 (9): 153 - 155.

4. Hickey AJ, Gounder L, Moosa MY, Drain PK. (2015). A systematic review of hepatic tuberculosis with considerations in human immunodeficiency virus co-infection. BMC Infectious Diseases 15 (1): 209.

5. Yu RS, Zhang SZ, Wu JJ, Li RF. (2004). Imaging diagnosis of 12 patients with hepatic tuberculosis. World Journal of Gastroenterology WJG 10 (11): 1639.

6. Cao BS, Li XL, Li N, Wang ZY. (2010). The nodular form of hepatic tuberculosis: Contrast-enhanced ultrasonographic findings with pathologic correlation. Journal of Ultrasound in Medicine 29 (6): 881 - 888.

7. El Bouazzi O, Hammi S, Bourkadi JE et al. (2016). First line anti-tuberculosis induced hepatotoxicity: incidence and risk factors. The Pan African Medical Journal 25: 167.

8. Chen HC, Chao YC, Shyu RY, Hsieh TY. (2003). Isolated tuberculous liver abscesses with multiple hyperechoic masses on ultrasound: a case report and review of literature. Liver Int 23: 346 - 350. 
